\title{
Small-animal whole-body imaging using a photoacoustic full ring array system
}

Jun Xia, Zijian Guo, Andres Aguirre, Quing Zhu, Lihong V. Wang

Jun Xia, Zijian Guo, Andres Aguirre, Quing Zhu, Lihong V. Wang, "Smallanimal whole-body imaging using a photoacoustic full ring array system," Proc. SPIE 7899, Photons Plus Ultrasound: Imaging and Sensing 2011, 789911 (17 February 2011); doi: 10.1117/12.873401

SPIE. Event: SPIE BiOS, 2011, San Francisco, California, United States 


\title{
Small-animal whole-body imaging using a photoacoustic full-ring array system
}

\author{
Jun Xia ${ }^{\mathrm{a}}$, Zijian Guo ${ }^{\mathrm{a}}$, Andres Aguirre ${ }^{\mathrm{b}}$, Quing Zhu ${ }^{\mathrm{b}}$, and Lihong V. Wang ${ }^{\mathrm{a} *}$ \\ ${ }^{a}$ Dept. of Biomedical Engineering, Washington University in St. Louis, St. Louis, MO 63130 USA \\ ${ }^{\mathrm{b}}$ Dept. of Electrical Engineering, University of Connecticut, Storrs, CT 06269 USA \\ *Correspondence should be addressed to LHWang@biomed.wustl.edu
}

\begin{abstract}
In this report, we present a novel 3D photoacoustic computed tomography (PACT) system for small-animal whole-body imaging. The PACT system, based on a 512-element full-ring transducer array, received photoacoustic signals primarily from a 2-mm-thick slice. The light was generated by a pulse laser, and can either illuminate from the top or be reshaped to illuminate the sample from the side, using a conical lens and an optical condenser. The PACT system was capable of acquiring an in-plane image in $1.6 \mathrm{~s}$; by scanning the sample in the elevational direction, a 3D tomographic image could be constructed. We tested the system by imaging a cylindrical phantom made of human hairs immersed in a scattering medium. The reconstructed image achieved an in-plane resolution of $0.1 \mathrm{~mm}$ and an elevational resolution of $1 \mathrm{~mm}$. After deconvolution in the elevational direction, the 3D image was found to match well with the phantom. The system was also used to image a baby mouse in situ; the spinal cord and ribs can be seen easily in the reconstructed image. Our results demonstrate that the PACT system has the potential to be used for fast small-animal whole-body tomographic imaging.
\end{abstract}

Keywords: Whole-body imaging, photoacoustic tomography, full-ring transducer array, spinal cord.

\section{INTRODUCTION}

Due to the widespread use of animal models for human diseases, animal imaging plays an important role in biomedical studies. Previously, the majority of small-animal whole-body imaging systems were based on radioactive techniques such as micro-computed tomography (micro-CT) and positron emission tomography (PET). However, the potentially harmful radiation prevents repeated usage of these techniques. The photoacoustic (optoacoustic) technique has recently emerged as an important tool for small-animal imaging [1]. By combining optical sensitivity and ultrasonic imaging depth scalability, this hybrid technology provides high-resolution images beyond the soft depth limit of conventional optical imaging technologies. Over the past few years, several array-based photoacoustic whole-body imaging systems have been proposed, including 64-element arc array [2, 3], 64-element half-ring array [4], and 128-element hemisphere array [5]. However, these systems have either limited spacial resolution or long data acquisition time. In this report, we present a novel whole-body imaging system based on a 512-element full-ring transducer array.

\section{SYSTEM DESIGN}

Figure 1 shows the schematic diagram of the system. The key components include a 512-element full-ring transducer array, a 64-channel data acquisition (DAQ) system, an optical parametric oscillator (OPO) laser, and a Ti:sapphire laser.

The 512-element full-ring transducer array (Imasonic, Inc.) has a center frequency of $5 \mathrm{MHz}$ and a reception bandwidth greater than $80 \%$. The piezocomposite elements are spaced with a lateral pitch of one wavelength $(0.3 \mathrm{~mm})$ and kerf of $0.1 \mathrm{~mm}$. The array element is cylindrically focused to form an imaging slice of about $2 \mathrm{~mm}$ thickness. In order to save the number of DAQ channels, 8:1 multiplexing is used. As such, a 512-element capturing requires 8 laser pulses [6].

The excitation source consists of two lasers. An optical parametric oscillator (OPO) laser system (Opotek, Inc.), with tunable wavelength from 400 to 680 and 740 to $2000 \mathrm{~nm}$, and a Ti:sapphire laser (Symphotic TII) tunable from 680 to $900 \mathrm{~nm}$. The combination of two lasers provides a solid coverage from 400 to $2000 \mathrm{~nm}$ for studying new optical

Photons Plus Ultrasound: Imaging and Sensing 2011, edited by Alexander A. Oraevsky, Lihong V. Wang, Proc. of SPIE Vol. 7899, 789911 - @ 2011 SPIE · CCC code: 1605-7422/11/\$18 · doi: 10.1117/12.873401 
contrasts. The system initially used top illumination for brain imaging [7]. For small-animal whole-body imaging, side illumination is introduced by using a conical lens and a lab-made optical condenser.

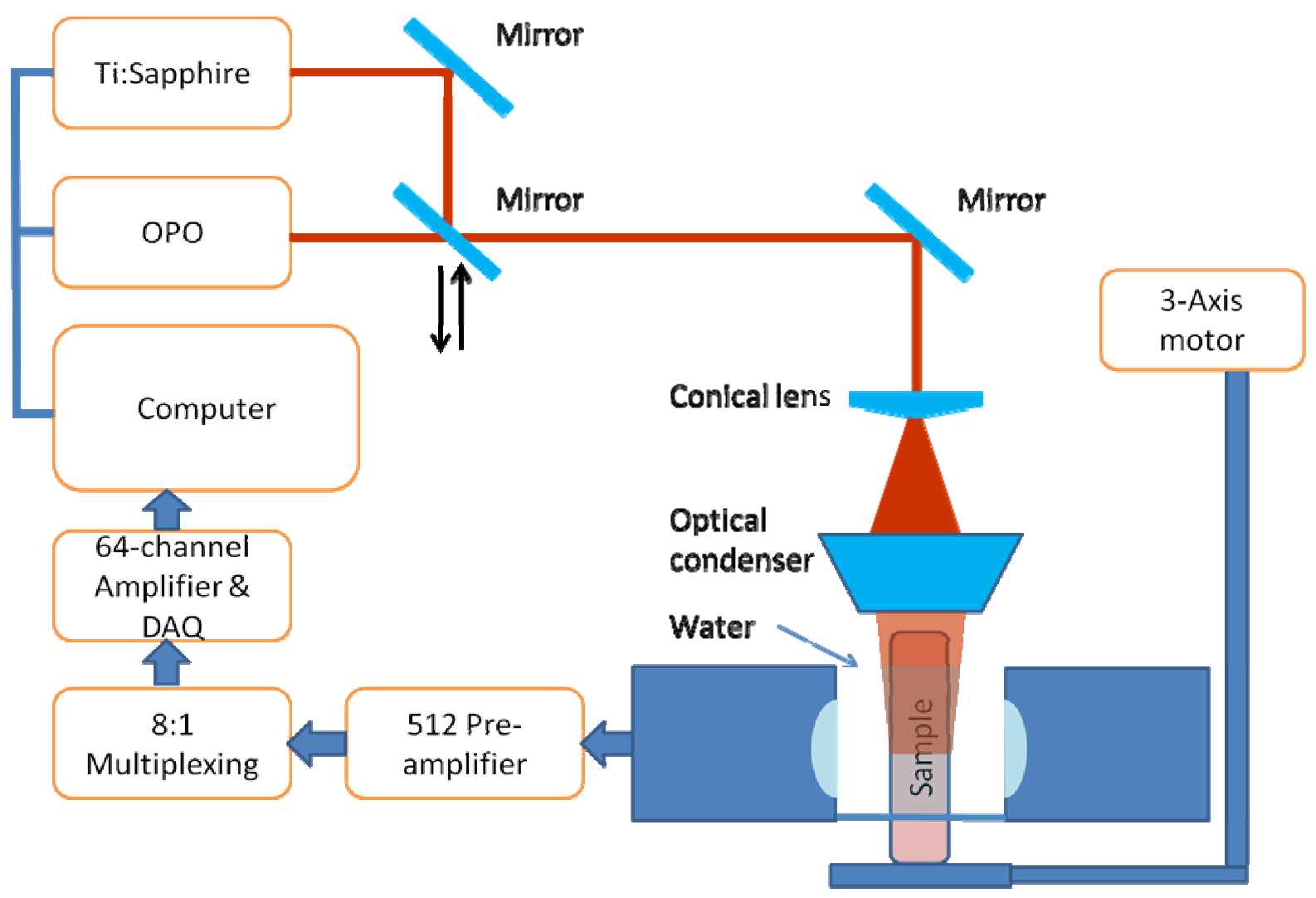

Figure 1. Schematic diagram of the full-ring whole-body scanning system.

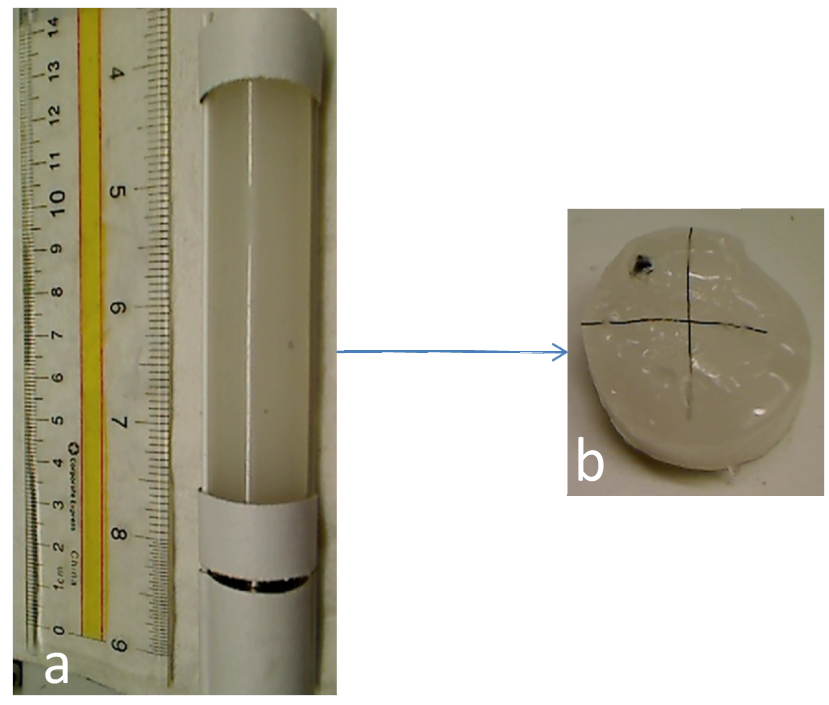

Figure 2. a. The tissue mimic crylindrical phantom made with $1 \%$ intralipid solution; $b$. The graphite and hairs embedded in the phantom. 


\section{RESULTS}

To validate the system performance, we first imaged a cylindrical phantom embedded with two crossing human hairs and a piece of graphite (Figure 2). By scanning the phantom for $8 \mathrm{~mm}$ along elevational direction, we obtained a 3D image shown in Figure 3a. Based on the image, we quantified the in-plane resolution to be $0.1 \mathrm{~mm}$ and the elevational resolution to be $1 \mathrm{~mm}$. Because our scanning range covers the whole imaging object, and the point distribution function is shift-invariant in elevation direction, we used the Richardson-Lucy deconvolution algorithm to improve the reconstructed image. Figure $3 b$ shows the deconvolved image, where the elevational resolution is improved up to 0.2 $\mathrm{mm}$.
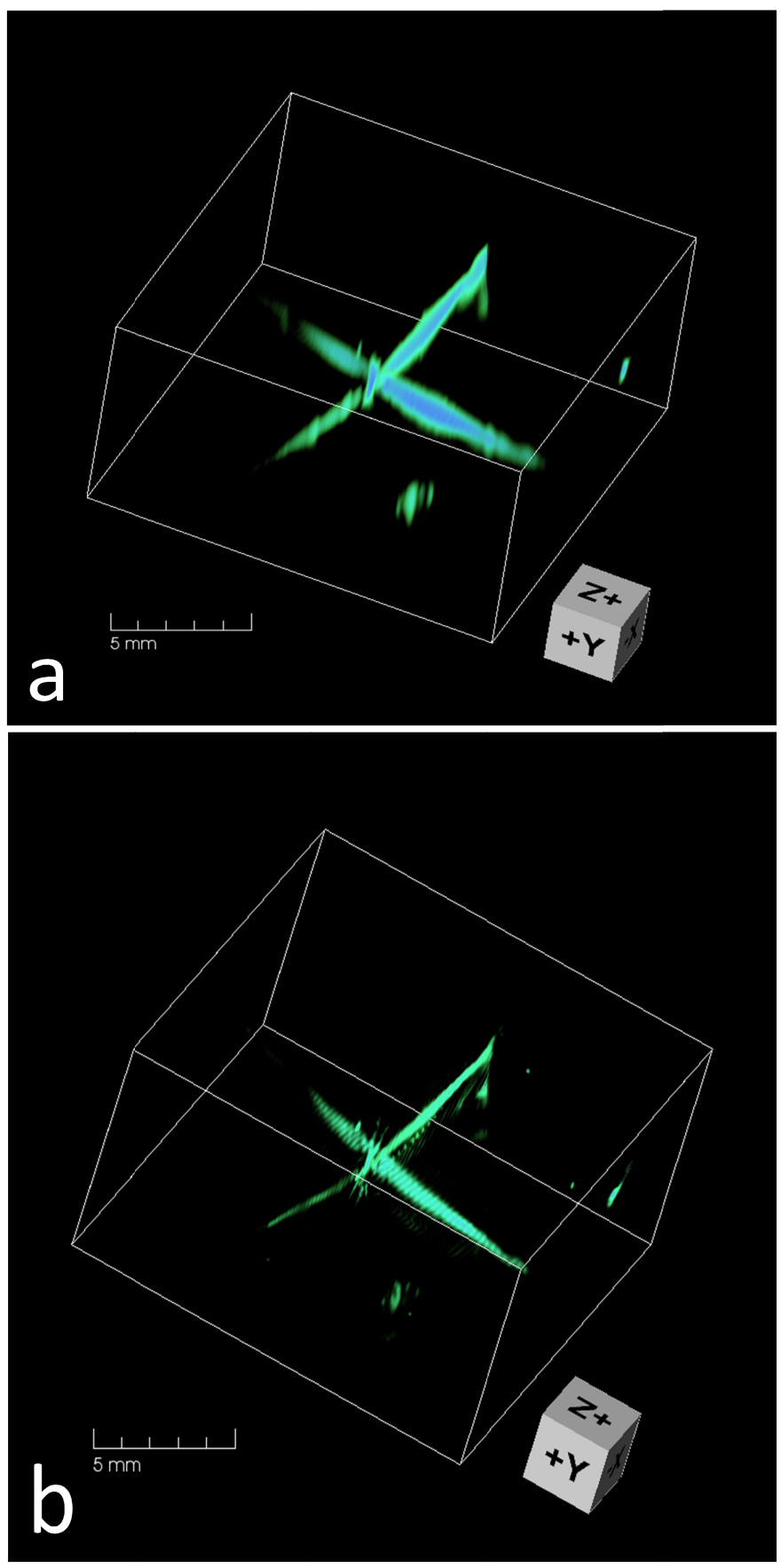

Figure 3. a. Reconstructed 3D photoacoustic image; b. Photoacoustic image after deconvolution in elevational direction. 
We also imaged a 1-day-old nude mouse in situ. In this experiment, we used the $532 \mathrm{~nm}$ wavelength from the OPO laser to illuminate from the top. The optical fluence $\left(10 \mathrm{~mJ} / \mathrm{cm}^{2}\right)$ was under the ANSI safety limit $\left(31 \mathrm{~mJ} / \mathrm{cm}^{2}\right)$. All the imaging procedures were conducted in compliance with the experimental protocols approved by Washington University in St. Louis. Figure 4a shows the maximum-amplitude-projected photoacoustic image, where most of the features can be easily identified.
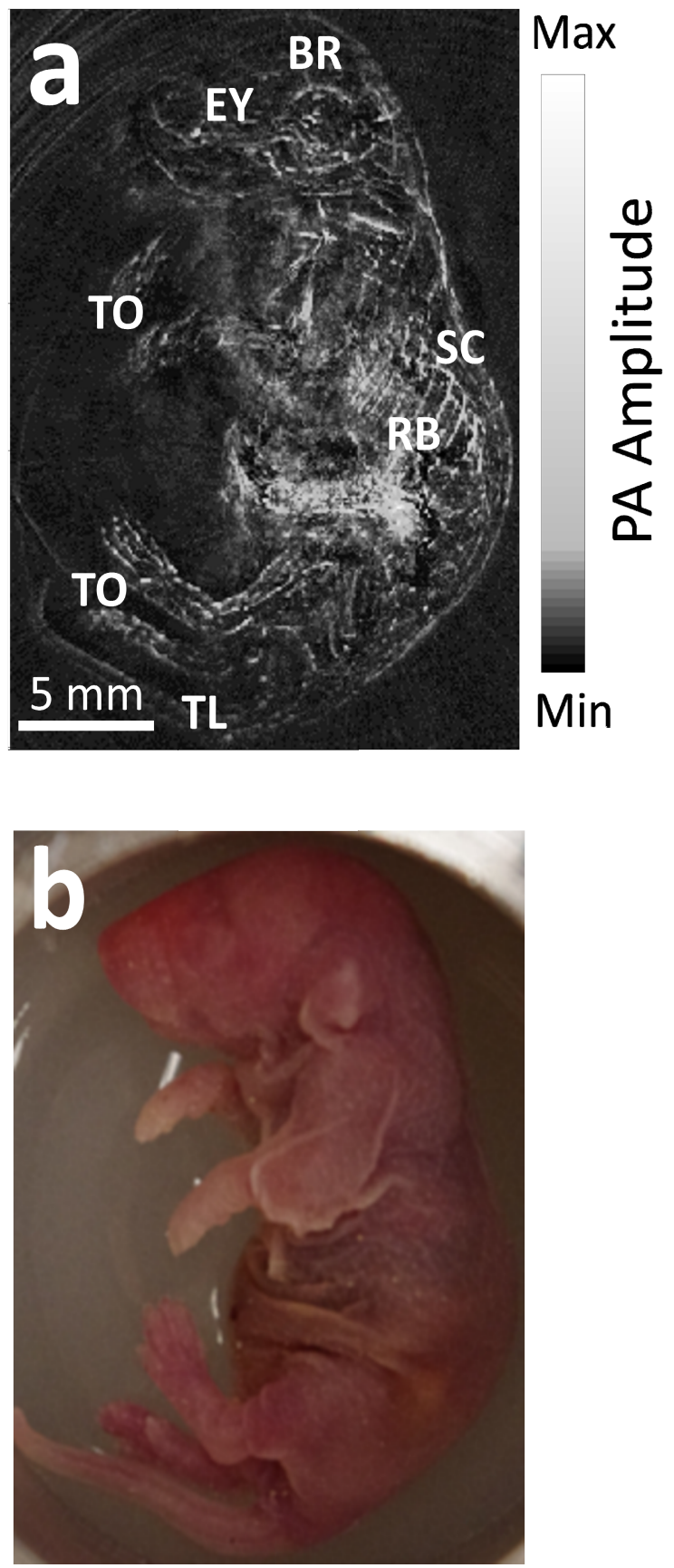

Figure 4. a. Maximum-amplitude-projected photoacoustic image of a 1-day-old nude mouse imaged at $532 \mathrm{~nm}$ laser wavelength; b. A photograph of the baby mouse. BR, brain; EY, eye; RB, rib; SC, spinal cord; TO, toes; TL, tail. 


\section{CONCLUSION}

A new small-animal whole-body imaging system has been developed based on the full-ring transducer array. The new system has an in-plane resolution of $0.1 \mathrm{~mm}$ and elevational resolution of $1 \mathrm{~mm}$. Using the Lucy-Richardson deconvolution algorithm, the elevational resolution can be further improved to $0.2 \mathrm{~mm}$. We also presented an in situ image of a baby mouse, where the spinal cord, ribs and major blood vessels can be seen easily.

\section{ACKNOWLEDGEMENTS}

This work was sponsored in part by National Institutes of Health grants R01 EB000712, R01 EB008085, R01 CA134539, U54 CA136398, R01 EB010049, and 5P60 DK02057933. L.W. has a financial interest in Microphotoacoustics, Inc. and Endra, Inc., which, however, did not support this work.

\section{REFERENCES}

[1] L. V. Wang, "Multiscale photoacoustic microscopy and computed tomography," Nature Photonics, 3(9), 503-509 (2009).

[2] H.-P. Brecht, R. Su, M. Fronheiser et al., "Whole-body three-dimensional optoacoustic tomography system for small animals," Journal of Biomedical Optics, 14(6), 064007-8 (2009).

[3] D. R. Reinecke, R. A. Kruger, R. B. Lam et al., "Co-registered photoacoustic, thermoacoustic, and ultrasound mouse imaging." 7564, 756420-9.

[4] A. Buehler, E. Herzog, D. Razansky et al., "Video rate optoacoustic tomography of mouse kidney perfusion," Opt. Lett., 35(14), 2475-2477 (2010).

[5] R. Kruger, D. Reinecke, G. Kruger et al., "HYPR-spectral photoacoustic CT for preclinical imaging," Proceedings of SPIE, 7177, 71770F (2009).

[6] J. Gamelin, A. Maurudis, A. Aguirre et al., "A real-time photoacoustic tomography system for small animals," Opt. Express, 17(13), 10489-10498 (2009).

[7] C. Li, A. Aguirre, J. Gamelin et al., "Real-time photoacoustic tomography of cortical hemodynamics in small animals," Journal of Biomedical Optics, 15(1), 010509-3 (2010). 